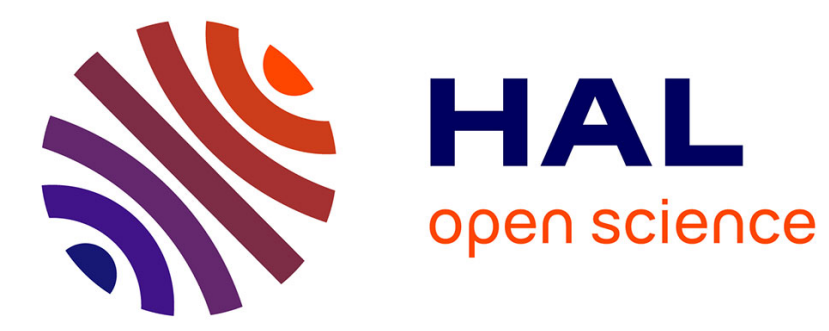

\title{
Systematic template extraction from chaotic attractors: I. Genus-one attractors with an inversion symmetry
}

Martin Rosalie, Christophe Letellier

\section{To cite this version:}

Martin Rosalie, Christophe Letellier. Systematic template extraction from chaotic attractors: I. Genus-one attractors with an inversion symmetry. Journal of Physics A: Mathematical and Theoretical, 2013, 46 (37), pp.375101. 10.1088/1751-8113/46/37/375101 . hal-01003677

\section{HAL Id: hal-01003677 https://hal.science/hal-01003677}

Submitted on 10 Aug 2016

HAL is a multi-disciplinary open access archive for the deposit and dissemination of scientific research documents, whether they are published or not. The documents may come from teaching and research institutions in France or abroad, or from public or private research centers.
L'archive ouverte pluridisciplinaire HAL, est destinée au dépôt et à la diffusion de documents scientifiques de niveau recherche, publiés ou non, émanant des établissements d'enseignement et de recherche français ou étrangers, des laboratoires publics ou privés. 


\title{
Systematic template extraction from chaotic attractors. I genus-one attractors with an inversion symmetry
}

\author{
Martin Rosalie \& Christophe Letellier \\ CORIA UMR 6614 - Université et INSA de Rouen, Av. de l'Université, BP 12, \\ F-76801 Saint-Etienne du Rouvray cedex, France \\ E-mail: martin.rosalie@coria.fr
}

\begin{abstract}
Describing the topological properties by a template is a powerful technique to classify chaotic attractors. Most of the time, reduced template are used but direct templates - in which all mechanisms (torsions, branch permutation, etc.) identified in the attractor are explicitely described without any simplification - are of a great interest to have a better description of the subtleties of the dynamics. We here introduce two additional conventions to represent in an unique way the reduced template from a given linking matrix. Then, we propose an addition law for linking matrices which allows to manipulate (combine) different mechanisms. Direct template can thus be described by using a series of linking matrices. In the other hand, we show how to analytically build the linking matrix associated with an attractor which is the image under an inversion symmetry of an attractor whose linking matrix is known.
\end{abstract}

PACS numbers: 05.45.-a

Submitted to: J. Phys. A: Math. Gen. 3 May 2013 


\section{Introduction}

Chaotic attractors produced by three-dimensional differential equations are of various types as well exemplified by the Rössler attractor [1], the Lorenz attractor [2], the double-scroll attractor $[3,4]$, the $\mathrm{Li}$ attractor $[5,6]$. Many works were devoted to their characterization by branched manifolds or templates (see $[7,8,9,10,11]$ among others). If for simple attractors as the Rössler attractor bounded by a genus-one torus [12], there is no problem for providing a template, there are many non trivial details which should be evoked for attractors produced, for instance, by systems with symmetry properties: this is the case of the Lorenz system which presents a rotation symmetry $\mathcal{R}_{z}(\pi)[13,14,15]$. The Lorenz attractor is bounded by a genus-3 torus [12] and the Poincaré section is made of two components as it was first pointed out by Letellier and co-workers [16].

Before investigating attractors bounded by large genus tori, we will show in this paper that some chaotic attractors bounded by genus-one tori are nontrivial and that the template is not necessarily unique depending on the location of the Poincaré section used as a reference to build it. Moreover, in most of the studies, only reduced templates are discussed, that is, the simplest variant of the template modulo Reidemeister moves. Nevertheless, it was recently shown that to determine whether an attractor was new or not, the so-called direct template must be considered [17]: the main argument was that the attractor produced by a flow inducing, for instance, a positive global torsion followed by a negative global torsion cannot be considered as associated with the same dynamics as an attractor solution to a flow without any global torsion. By global torsion, we designate here a torsion applied to all the branches which can be distinguished in a template. A side problem is that the corresponding (direct or reduced) template is not unique when there are global torsions. We will therefore develop a procedure for an explicit description of direct templates as well as the relationship between the linking matrices describing the different templates which can be obtained for a single attractor. In order to do that, the attractors produced by the simplest equivariant jerk system proposed by Malasoma [18] are used.

The subsequent part of this paper is organized as follows. In Section 2, templates and how they are described by linking matrices are introduced. Section 3 is devoted to the topological analysis of a chaotic attractor for which different templates can be obtained depending on the Poincaré section used as a reference. The system that produces the bench attractor is introduced. Direct and reduced templates are then constructed for which some specific rules to sum linking matrices are introduced. Section 4 discusses how the symmetric companion of an attractor under an inversion symmetry can be topologically characterized from the linking matrix obtained for the initial attractor. Section 5 gives a conclusion.

\section{Template and linking matrix}

A template or a branched manifold is a branched surface that synthesizes the topology of an attractor by describing the relative organisation of the population of unstable periodic orbits [19] which constitutes the skeleton of the attractor [10]. Any template for a chaotic attractor bounded by a genus-one torus can be divided in two main parts (Fig. 1): i) a part that describes the dynamical processes (stretching, folding, squeezing, etc.) responsible for the mixing properties of the chaotic trajectories and their sensitivity to initial conditions and, ii) a trivial part where segments of 
trajectories are everywhere locally parallel. The first part is here named a "mixer" for designating the overall mechanism which has an action on the branches that can be distinguished in a chaotic attractor: these branches are determined, for instance, according to the critical points of a first-return map to a Poincaré section when the system is dissipative enough to present a one-dimensional Poincaré map [8]. A mixer results from some basic processes applied to the branches (torsion, permutation, stretching, squeezing, etc.). When stretching and squeezing are absent, it is also possible to have an action on the branches which does not lead to chaos as we will detail below. We therefore introduce the concept of linker for designating torsions, branch permutations while stretching and squeezing mechanisms are grouped into the branch insertion as proposed by Tufillaro and co-workers [7]. A linker can be made of few linkers. For instance, the linker between the splitting chart and the branch insertion shown in Fig. 1a is made of one linker for the local torsion (applied to the right branch) and one linker for the branch permutation. In general, we will use a single linker (which is also a mixer in such a case) to describe the template shown in Fig. 1a.

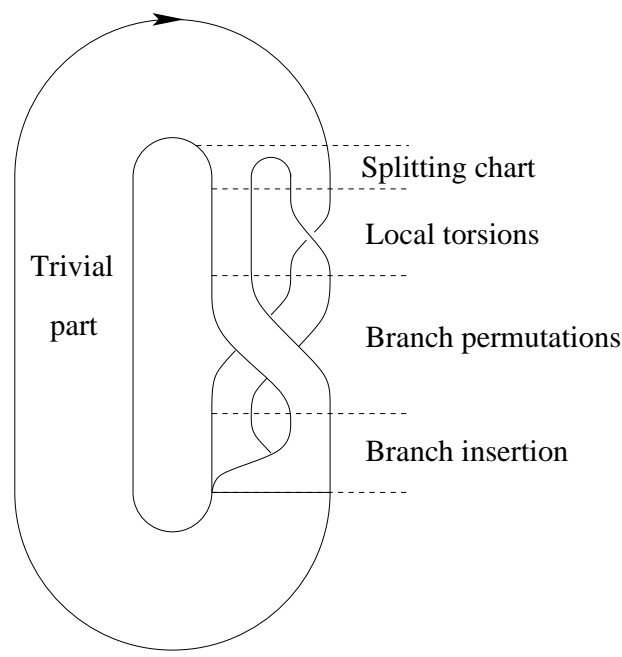

(a) "Chaotic" template

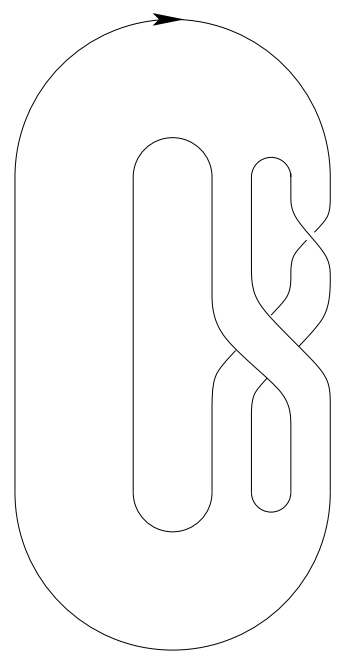

(b) "Non chaotic" template

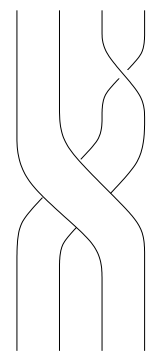

(c) Open linker

Figure 1. A typical template and the way in which it is divided into two parts, and two of its variants (see the main text).

A linker can be encoded by a so-called linking matrix $L$. Element $L_{i i}$ corresponds to the number of signed $\pi$-twists the $i$ th branch presents, and element $L_{i j}$ is equal to the number of signed crossings (or permutations) between the $i$ th and the $j$ th branches. All elements are signed according to the convention shown in Fig. 2. Torsions are expressed in terms of signed $\pi$-twists: thus, a $\alpha$-torsion refers to a $\alpha \cdot \pi$ twist $(\alpha \in \mathbb{Z})$. There are two equivalent representations for describing a template with a linking matrix. One, introduced by Mindlin et al. [20], is composed by a linking matrix associated with a vector that indicates the order with which the branches are inserted from the back to the front. One of the problem we have with this notation is that it is not possible to make direct comparison between two templates by only comparing the linking matrices. To overcome this problem Cross and Gilmore used a dressed return map to isolate the mechanism responsible for the chaotic nature of the 
solution [21]. The second representation, introduced by Tufillaro and coworkers [7], is based on a standard insertion convention according to which branches are ordered from the left (back) to the right (front).

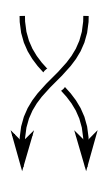

(a) Positive torsion

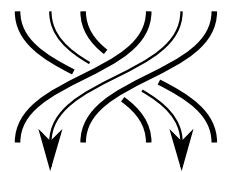

(c) Positive permutation

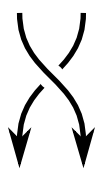

(b) Negative torsion

(d) Negative permutation

Figure 2. Sign convention for $\pi$-twist ors permutations. The arrows indicate the direction of the flow.

Since the standard insertion convention allows to compare different templates using the sole linking matrix, we retained this second representation. To avoid any ambiguity, we will add another convention which constrains the back branch in the branch insertion to be the closest branch to the singular point surrounded by the flow. This additional convention avoids many equivalent templates for a single linking matrix. Moreover, also to avoid various equivalent templates, we will always use a representation of the attractor with a clockwise flow, as used in the bounding tori theory [12]. These additional conventions are required to ensure the uniqueness of the template drawn from a given linking matrix.

In the template shown in Fig. 1, the non trivial part of the flow corresponds to the mechanisms which are responsible for the chaotic nature of the solution. These mechanisms can be schemed as follows.

(a) the stretching and the folding processes which are most often concomitant;

(b) the squeezing mechanism for inserting the branches into a single one.

Then the trivial part of the flow undergoes to the next splitting chart. In a similar way although more schematic, the non trivial part of the template can be divided as follows.

(i) a splitting chart [7] which allows to distinguish the different branches;

(ii) the local torsion in each branch corresponding to elements $L_{i i}$;

(iii) the permutation between the branches corresponding to elements $L_{i j}(i \neq j)$;

(iv) the insertion where the branches are stretched and squeezed into a single global branch.

Then follows the trivial part of the template. Dynamically speaking, the splitting chart does not contain any information. This is a "virtual" split introduced for the clarity of the template. The branch insertion encodes the main features for producing chaotic solutions, that is, the stretching and the squeezing mechanisms. These two mechanisms are responsible for returning the $n$ branches distinguished by the splitting chart into a single large branch corresponding to the trivial part of the template. Without branch insertion, there is no chaos. Indeed, if a linker is ended with a "branch 
merging" (Fig. 1b) rather than with a branch insertion (Fig. 1a), only periodic orbits can be obtained. The branch insertion is therefore relevant for chaos production. The first template (Fig. 1a) is characterized by a linker with a branch insertion, that is, by a mixer, while the second (Fig. 1b) is not because the branch insertion was replaced with a branch merging. For this reason, we will have to take into account the branch insertion in our linking matrices theory as explained in the subsequent part of this paper.

By itself, a linking matrix only encodes mechanisms (ii) and (iii) of the template, the branch insertion being implicitly encoded by the standard insertion convention. In the subsequent part of this paper, and as detailed in the two examples shown in Figs. 1, it will be useful to distinguish a linking matrix without branch insertion from a linking matrix with a branch insertion. As we mentioned, the splitting chart is only virtual and is useful for designating the end of the trivial part and the beginning of a linker. It will be designated by a simple bracket "[". The linking matrix of a linker terminated by a "branch merging" (by opposition to a branch insertion) will be closed by a right bracket "]". Thus, the linker shown in Fig. 1b is associated with the linking matrix

$$
L_{\cap \cup}=\left[\begin{array}{cc}
0 & -1 \\
-1 & -1
\end{array}\right]
$$

where indices $\cap$ and $\cup$ designate the splitting chart and the branch merging, respectively. When a branch insertion replaces the branch merging as shown in Fig. 1a, the linking matrix becomes

$$
L_{\cup \mid}=\left[\begin{array}{cc}
0 & -1 \\
-1 & -1
\end{array}\right]
$$

that is, the right bracket is replaced with a double bracket "】". The last case to consider corresponds to a linker inserted in a series of linkers: as a consequence there is no splitting chart nor branch merging as shown in Fig. 1c. The linking matrix is in the latter case written as

$$
L=\left|\begin{array}{cc}
0 & -1 \\
-1 & -1
\end{array}\right|
$$

where the brackets are replaced with simple bars. This type of linker will be designated as an "open" linker. In the case where there is a single bracket (single or double), the linker is thus semi-open, or semi-closed.

Let us consider the case of global torsion - which designates in this work a torsion applied to all branches. Fig. 3a corresponds to a non regular representation of a positive global 1-torsion because more than two segments of the representation cross at the same point. Fig. 3b corresponds to a regular representation of the same positive 1-torsion. The corresponding linking matrix is thus

$$
T=\left|\begin{array}{ll}
+1 & +1 \\
+1 & +1
\end{array}\right|
$$

When the linker starts with a splitting chart and ends with a branch insertion (Fig. $3 \mathrm{c})$, the linking matrix is

$$
T_{\cup \mid}=\left[\begin{array}{ll}
+1 & +1 \\
+1 & +1
\end{array}\right] .
$$


This closed linker is a mixer and could produce chaotic solution. To the best of our knowledge, there is not yet a continuous flow corresponding to such a template. The corresponding attractor should be characterized by a first-return map to a Poincaré section with two increasing monotonic branches (a flow with such a map was obtained by Rössler [22] but the two branches have local torsions which differ by $2 \pi$ ). The last case shown in Fig. 3d is obtained by replacing the branch insertion by a branch merging: it is thus described by the linking matrix

$$
T_{\mathrm{n} \cup}=\left[\begin{array}{ll}
+1 & +1 \\
+1 & +1
\end{array}\right] .
$$

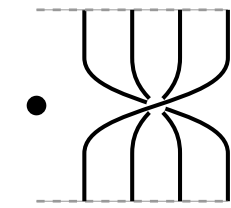

(a) Non regular

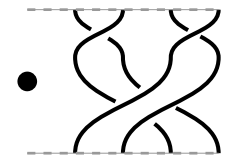

(b) Regular

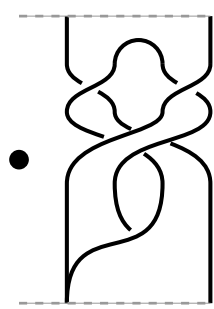

(c) With splitting and insertion

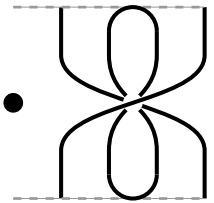

(d) With splitting and merging

Figure 3. A positive global 1-torsion (a) applied to two branches. A regular representation (b) clearly distinguishes the local torsion (in each branch) from the permutation between the two branches. The positive global torsion is also drawn with the splitting chart and the branch insertion (c). By convention, the singular point • surrounded by the flow is always located at the left of the non trivial part of the template. In (c), the back branch is closer to the singular point than the front branch. In (d), the linker starts with a splitting chart and ends with a branch merging.

\section{Topological analysis of a non trivial attractor}

\subsection{The simplest equivariant jerk system}

The simplest equivariant jerk system

$$
\dddot{x}=-\alpha \ddot{x}+x \dot{x}^{2}-x
$$

was introduced by Malasoma [18]. It can be rewritten as the set of three differential equations

$$
\left\{\begin{array}{l}
\dot{x}=y \\
\dot{y}=z \\
\dot{z}=-\alpha z+x y^{2}-x,
\end{array}\right.
$$

where $y=\dot{x}$ and $z=\ddot{x}$. This system is equivariant, that is, it obeys to the relation $\Gamma \cdot \boldsymbol{f}(\boldsymbol{x})=\boldsymbol{f}(\Gamma \cdot \boldsymbol{x})$ where

$$
\Gamma=\left[\begin{array}{ccc}
-1 & 0 & 0 \\
0 & -1 & 0 \\
0 & 0 & -1
\end{array}\right]
$$

is a matrix defining the symmetry properties, that is, an inversion symmetry in the present case [23]. The system (8) has a single singular point located at the origin of the 
phase space. For $\alpha=2.0645$ and from the initial conditions $\boldsymbol{x}_{0}=\left(x_{0}, y_{0}, z_{0}\right)=(+4,0,0)$, the trajectory settles down onto the chaotic attractor $\mathcal{A}$ shown in Fig. 4. This attractor is asymmetric, that is, there is a companion attractor $\overline{\mathcal{A}}$ from the initial conditions $\Gamma \cdot \boldsymbol{x}_{0}$ which coexists in the phase space. The companion attractor $\bar{A}$ will be investigated in Section 4.

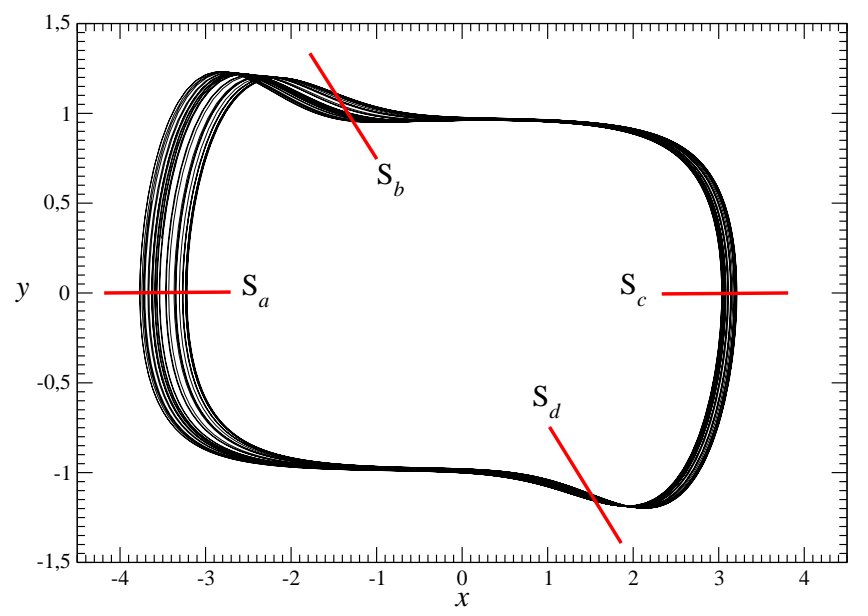

Figure 4. Asymmetric chaotic attractor $\mathcal{A}$ solution to the system (8) for $\alpha=2.0645$. The flow is clockwise. Different Poincaré sections are also represented.

The attractor solution to the simplest equivariant jerk system is bounded by a genus-one torus. The Poincaré section is therefore made of a single component [12]. In principle, the component of the Poincaré section can be equivalently chosen as any transverse section to the flow, transverse meaning non tangent to the flow. From Poincaré's works [24], the component of the Poincaré section is defined by a half-plane containing the singular point surrounded by the flow: such a constraint avoids any tangency between the flow and the Poincaré section. In Fig. 4, we drew four possible Poincaré sections defined by four angles $\varphi_{k}$ in the $x-y$ plane. According to our choices, the four Poincaré sections are defined as $\mathrm{S}_{a}, \mathrm{~S}_{b}, \mathrm{~S}_{c}$ and $\mathrm{S}_{d}$ corresponding to the angles $\varphi_{a}=\pi, \varphi_{b}=\frac{4 \pi}{5}, \varphi_{c}=0$, and $\varphi_{d}=\frac{9 \pi}{5}$, respectively. The flow thus visits the sections $\mathrm{S}_{a}, \mathrm{~S}_{b}, \mathrm{~S}_{c}$ and $\mathrm{S}_{d}$, successively.

Once the Poincaré section is chosen, a first-return map is constructed. In the present case, we used the cylindrical coordinates applied to the $x$ - $y$ plane. Thus, any point $(x, y, z)$ is investigated using variables $(r, \theta, z)$. The Poincaré sections are thus defined as

$$
\mathcal{P}_{i} \equiv\left\{\left(r_{n}, z_{n}\right) \in \mathbb{R}^{2} \mid \theta_{n}=\varphi_{i}, \dot{\theta}_{n}<0\right\} .
$$

For each of the four Poincaré sections, the first-return map is shown in Fig. 5. The four first-return maps are smooth and unimodal. Nevertheless, the two monotonic branches of the first-return maps to Poincaré section $\mathrm{S}_{a}$ and $\mathrm{S}_{d}$ are split by a maximum (Figs. $5 \mathrm{a}$ and $5 \mathrm{~d}$ ) and by a minimum for Poincaré sections $\mathrm{S}_{b}$ and $\mathrm{S}_{c}$ (Figs. $5 \mathrm{~b}$ and $5 \mathrm{c}$ ). This means that there is an odd global torsion between sections $S_{a}$ and $S_{b}$ and one between sections $\mathrm{S}_{c}$ and $\mathrm{S}_{d}$. Up-to-now, no difference was made in the treatment of these two types of first-return maps; using the property that all Poincaré sections are equivalent, the axes were implicitly inverted in order to recover the critical point dividing the two monotonic branches located at the maximum of the map [8]. Nevertheless, in two 
cases, the increasing branch is closer to the singular point surrounded by the flow than the decreasing branch, and in the two others, it is not.

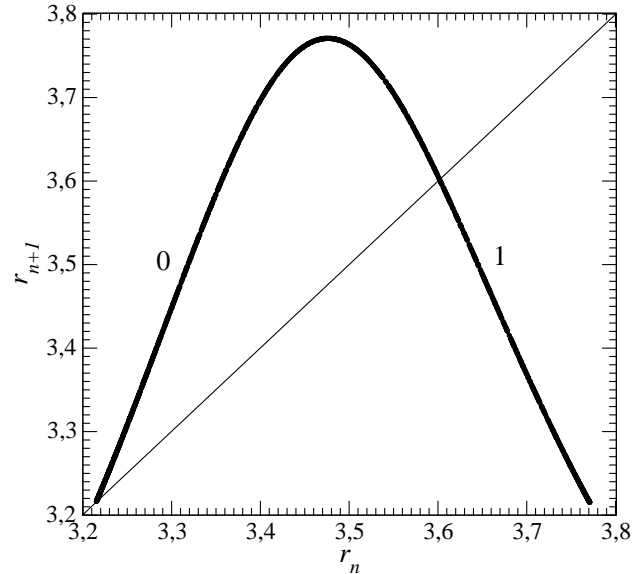

(a) Section $\mathrm{S}_{a}$

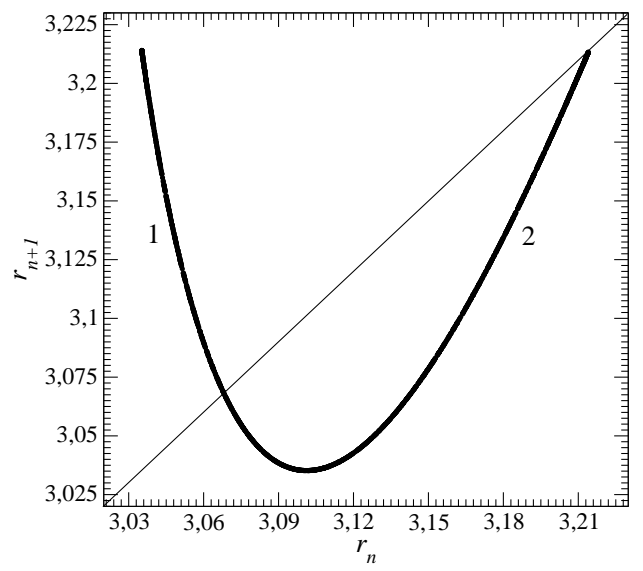

(c) Section $\mathrm{S}_{c}$

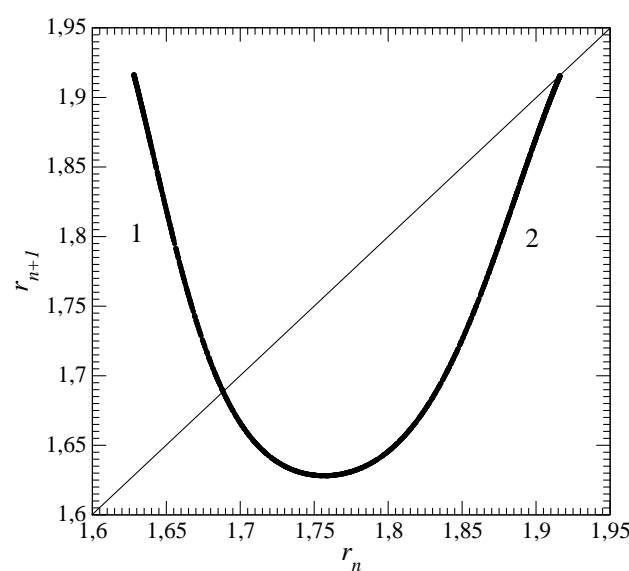

(b) Section $\mathrm{S}_{b}$

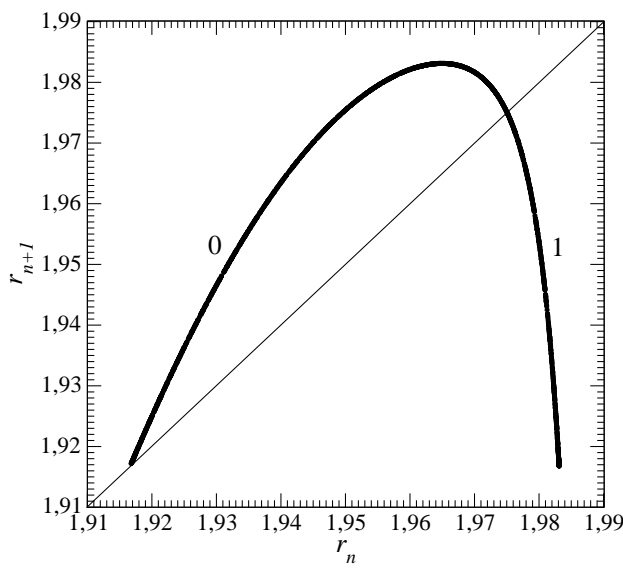

(d) Section $\mathrm{S}_{d}$

Figure 5. First-return maps for the four Poincaré sections of the asymmetric chaotic attractor solution $\mathcal{A}$ to system (8). Parameter value: $\alpha=2.0645$.

\subsection{Reduced template}

Depending on the Poincaré section chosen as a reference, the symbolic dynamics is built on different symbols. Indeed, for working in sections $\mathrm{S}_{a}$ and $\mathrm{S}_{d}$ the symbolic dynamics is built on the symbols $\{0 ; 1\}$. Symbols $\{1 ; 2\}$ are used for working in the Poincaré sections $S_{b}$ and $S_{c}$. Odd (even) symbols are associated with decreasing (increasing) branches, and the natural order is followed from the singular point to the external boundary of the attractor. Parity of the symbols must match to the sign of the slope of monotonic branch as required to use the kneading theory [25, 26]. Note that results obtained by using symbols "0" and " 1 " and, then inverting the order of the symbolic sequences, are equivalent to those obtained with symbols "1" and "2".

The unstable periodic orbits are then extracted from the attractor and encoded. 
Linking numbers between various pairs of periodic orbits are computed as the halfsum of the oriented crossings counted in a regular plane projection [7]. The results are reported in Tab. 1 for the orbits encoded on $\{0 ; 1\}$. A similar table would have been obtained with the orbits encoded on $\{1 ; 2\}$ : to obtain it, it is sufficient to replace any "0" by a " 2 ". Since replacing " 0 " with " 2 " allows to switch from one symbolic dynamics to the other, the orbits are the same, and so the corresponding linking numbers.

Table 1. Linking numbers computed between the unstable periodic orbits extracted from the asymmetric chaotic attractor $\mathcal{A}$ solution to system (8). Parameter value: $\alpha=2.0645$.

\begin{tabular}{|c|c|c|c|c|c|c|}
\hline & (1) & (10) & (101) & (100) & (1011) & (1001) \\
\hline (10) & -1 & & & & & \\
\hline (101) & -1 & -2 & & & & \\
\hline$(100)$ & -1 & -2 & -3 & & & \\
\hline (1011) & -2 & -3 & -4 & -4 & & \\
\hline (1001) & -1 & -2 & -3 & -3 & -4 & \\
\hline (1000) & -1 & -2 & -3 & -3 & -4 & -4 \\
\hline
\end{tabular}

To predict these linking numbers, two templates can be proposed (Figs. 6). One (Fig. 6a) corresponds to the symbolic dynamics built on $\{0 ; 1\}$, and the other (Fig. $6 \mathrm{~b})$ to the symbolic dynamics built on $\{1 ; 2\}$, that is, using the Poincaré section $\mathrm{S}_{a}$ or $\mathrm{S}_{d}$ and $\mathrm{S}_{b}$ or $\mathrm{S}_{c}$ as a reference, respectively. These two templates are topologically equivalent since one can be transformed into the other using an isotopy by sending the external boundary (with the arrow in Figs. 6) close to the singular point surrounded by the flow. The mixer - here including a splitting chart and a branch insertion is then viewed from the back rather than from the front. These templates are reduced in the sense that there is no trace left from the global torsions we identified between sections $S_{a}$ and $S_{b}$, and between $S_{c}$ and $S_{d}$ : due to their opposite signs (see next section), they can be removed using Reidemeister moves. The important aspect of the "reduced" template approach is that the template is not unique and that there is no trace left of the two global torsions. The linking matrix is

$$
L_{\mathcal{A}_{a}}=L_{\mathcal{A}_{d}}=\left[\begin{array}{cc}
0 & -1 \\
-1 & -1
\end{array}\right],
$$

for the template shown in Fig. 6a, and

$$
L_{\mathcal{A}_{b}}=L_{\mathcal{A}_{c}}=\left[\begin{array}{cc}
-1 & -1 \\
-1 & 0
\end{array}\right],
$$

for the template shown in Fig. 6b. Here $\mathcal{A}_{a}$ designates the attractor $\mathcal{A}$ described by the template built starting from the Poincaré section $\mathrm{S}_{a}$, and $\mathcal{A}_{b}, \mathcal{A}_{c}$ and $\mathcal{A}_{d}$ for attractors built from sections $\mathrm{S}_{b}, \mathrm{~S}_{b}$ and $\mathrm{S}_{d}$, respectively. The corresponding mixers will be designated by $\mathcal{M}_{\mathcal{A}_{a}}, \mathcal{M}_{\mathcal{A}_{b}}, \mathcal{M}_{\mathcal{A}_{c}}$, and $\mathcal{M}_{\mathcal{A}_{d}}$, and their linking matrices by $L_{\mathcal{A}_{a}}, L_{\mathcal{A}_{b}}, L_{\mathcal{A}_{c}}$ and $L_{\mathcal{A}_{d}}$, respectively. The first matrix is commonly used to describe the topology of the spiral Rössler attractor [8]. The asymmetric chaotic attractor $\mathcal{A}$ 
solution to the simplest equivariant jerk system is therefore topologically equivalent to the spiral Rössler attractor. If the mechanism responsible of the chaotic nature of these two attractors are the same in the sense developed by Cross and Gilmore [21], these two attractors cannot be considered as being associated with the same type of dynamics since one has additional torsions compared to the other. This is one of the reasons for which the direct template was introduced in [17].

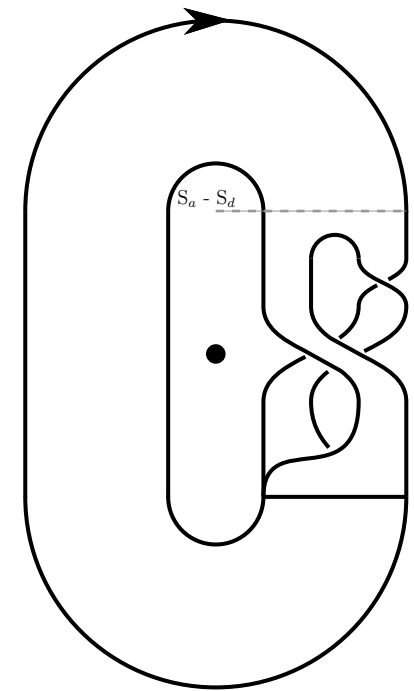

(a) From section $\mathrm{S}_{a}$ or $\mathrm{S}_{d}$

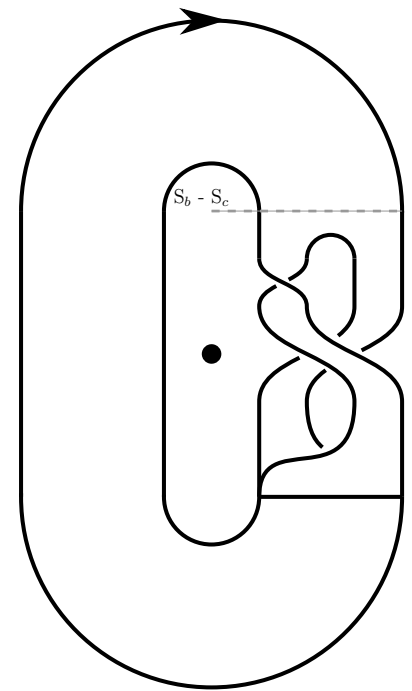

(b) From section $\mathrm{S}_{b}$ or $\mathrm{S}_{c}$

Figure 6. Two topologically equivalent templates for the asymmetric chaotic attractor $\mathcal{A}$ solution to system (8). Parameter value: $\alpha=2.0645$.

\subsection{Direct template}

A direct template [17] is a template describing all global and local torsions which can be identified in the corresponding flow. No simplification using Reidemeister moves is allowed. As deduced from the orientations of the four first-return maps shown in Figs. 5 and from the oriented crossings between segments of a trajectory, the direct template of the asymmetric attractor $\mathcal{A}$ solution to the simplest equivariant jerk system presents one negative 1-torsion between sections $S_{a}$ and $S_{b}$ and one positive 1-torsion between sections $\mathrm{S}_{c}$ and $\mathrm{S}_{d}$. A direct template is shown in Fig. 7a where, starting from section $\mathrm{S}_{a}$, there is one negative 1-torsion, one linker and one positive 1-torsion, successively.

Let us move the representation of the splitting chart (located after the negative 1-torsion in Fig. 7a) just before section $\mathrm{S}_{a}$ and the branch insertion at section $\mathrm{S}_{d}$ : we thus obtain the direct template shown in Fig. 7b. The direct template shown in Fig. $7 \mathrm{~b}$ is drawn in a regular representation. Moreover, all linkers are described with the same number of branches, a property which will be useful to manipulate their corresponding linking matrices. Thus, the template shown in Fig. $6 \mathrm{~b}$ is described by the sum of three linking matrices as

$$
L_{\mathcal{A}_{a}}=\underbrace{\left[\begin{array}{ll}
-1 & -1 \\
-1 & -1
\end{array} \mid\right.}_{\text {negative 1-torsion }}+\underbrace{\left|\begin{array}{cc}
0 & -1 \\
-1 & -1
\end{array}\right|}_{\text {linker }}+\underbrace{\left.\mid \begin{array}{ll}
+1 & +1 \\
+1 & +1
\end{array}\right]}_{\text {positive 1-torsion }}=\left[\begin{array}{cc}
0 & -1 \\
-1 & -1
\end{array}\right]
$$


All linking matrices are written in such a way that the element $L_{11}$ corresponds to the first branch (from the left to the right) at the splitting chart and the element $L_{22}$ is associated with the second branch. The central linker is open according to our definition and the two global torsions are associated with two semi-open linkers. The resulting linking matrix is "closed", that is, started with a splitting chart and ended with a branch insertion: this is thus a mixer.

Starting from the Poincaré section $\mathrm{S}_{b}$ or $\mathrm{S}_{c}$ changes the resulting linking matrix since the order of the branches is permuted compared to the previous description. For instance, starting from section $\mathrm{S}_{b}$ (Fig. 7b) leads to the linking matrix

$$
L_{\mathcal{A}_{b}}=\underbrace{\left[\begin{array}{cc}
-1 & -1 \\
-1 & 0
\end{array} \mid\right.}_{\text {linker }}+\underbrace{\left|\begin{array}{ll}
+1 & +1 \\
+1 & +1
\end{array}\right|}_{\text {positive 1-torsion }}+\underbrace{\left.\mid \begin{array}{ll}
-1 & -1 \\
-1 & -1
\end{array}\right]}_{\text {negative 1-torsion }}=\left[\begin{array}{cc}
-1 & -1 \\
-1 & 0
\end{array}\right] .
$$

Only the matrix describing the first linker is changed by switching from $\mathrm{S}_{a}$ to $\mathrm{S}_{b}$ as a result of the odd global torsion that permuted the order of the two branches. The matrix describing the linker is thus permuted. It will be thus convenient to introduce the permuted matrix $M^{p}$ of a given matrix $M$ defined as follows

$$
M^{p}=\left[\begin{array}{ccccc}
M_{n n} & M_{n n-1} & \ldots & M_{n 2} & M_{n 1} \\
M_{n-1} & M_{n-1} n-1 & \ldots & M_{n-12} & M_{n-1} \\
\vdots & \vdots & \ddots & \vdots & \vdots \\
M_{2 n} & M_{2 n-1} & \ldots & M_{22} & M_{21} \\
M_{1 n} & M_{1 n-1} & \ldots & M_{12} & M_{1} 1
\end{array}\right] .
$$

The linking matrices $L$ and $L^{p}$ describe two branched manifolds which are topologically equivalent. Combining a linker $\mathcal{L}$ with a global torsion $\mathcal{T}_{\eta}$ by $\eta$-torsion can be thus expressed according to

$$
\mathcal{L}^{\prime}=\mathcal{T}_{\eta}+\mathcal{L} \equiv \mid \begin{array}{ll}
T_{\eta}+L & \text { if } \eta \text { is even } \\
T_{\eta}+L^{p} & \text { if } \eta \text { is odd }
\end{array}
$$

where $L^{\prime}$ corresponds to the linking matrix describing a global $\eta$-torsion $\mathcal{T}_{\eta}$ associated with the linking matrix $T_{\eta}$ followed by a linker $\mathcal{L}$ corresponding to the linking matrix $L$. By definition, $T^{p}=T$, since any torsion is defined by $T=\eta \mathbb{I}$ where $\mathbb{I}$ is the unit matrix and, obviously, $\mathbb{I}^{p}=\mathbb{I}$. The two linking matrices (13) and (14) prove that there are two possible templates to describe the asymmetric chaotic attractor $\mathcal{A}$ shown in Fig. 4. The linking matrix (14) is also obtained when the template is built starting from section $\mathrm{S}_{c}$. In this case, the linking matrix is

$$
L_{\mathcal{A}_{c}}=\underbrace{\left[\begin{array}{ll}
+1 & +1 \\
+1 & +1
\end{array} \mid\right.}_{\eta=0}+\underbrace{\left|\begin{array}{ll}
-1 & -1 \\
-1 & -1
\end{array}\right|}_{\eta=-1}+\underbrace{\left.\mid \begin{array}{cc}
-1 & -1 \\
-1 & 0
\end{array}\right]}_{\text {linker }}=\left[\begin{array}{cc}
-1 & -1 \\
-1 & 0
\end{array}\right] .
$$

Note that starting from section $\mathrm{S}_{d}$ is rigorously equivalent to start from section $\mathrm{S}_{a}$ since the flow is "trivial" between these two Poincaré sections. We have thus

$$
L_{\mathcal{A}_{a}}=L_{\mathcal{A}_{b}}^{p}=L_{\mathcal{A}_{c}}^{p}=L_{\mathcal{A}_{d}} .
$$


There is thus no reason to prefer one template to the other. Both are topologically equivalent.

The advantage of direct template is that it clearly shows the difference between the asymmetric chaotic attractor $\mathcal{A}$ solution to the simplest equivariant jerk system and the spiral attractor solution to the Rössler system. This is particularly important for developing a classification of attractors. From our point of view, the asymmetric attractor $\mathcal{A}$ and the Rössler attractor should not be put in the same class, as clearly shown by the direct template since there are two additional global torsions in the former. This is here an additional evidence of the interest of direct template as introduced in [17].

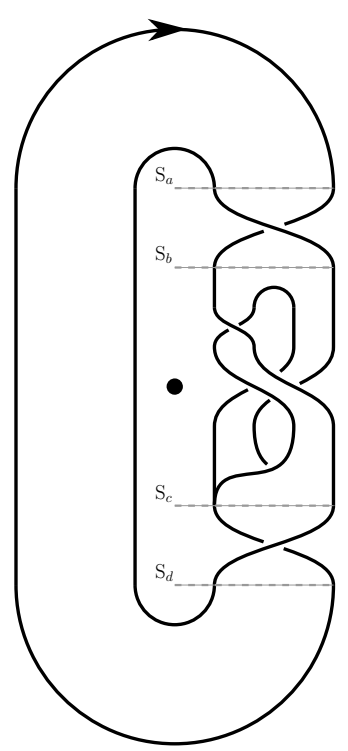

(a) Direct template from $\mathrm{S}_{a}$

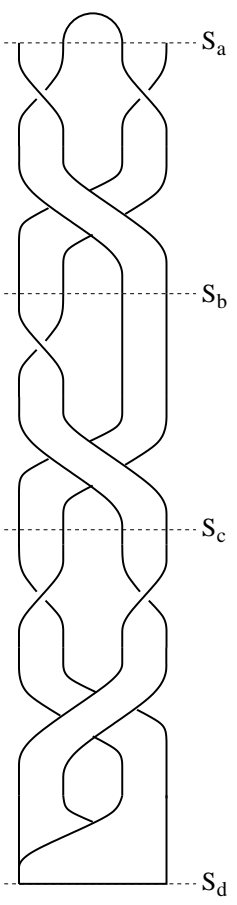

(b) Regular direct mixer from $\mathrm{S}_{a}$

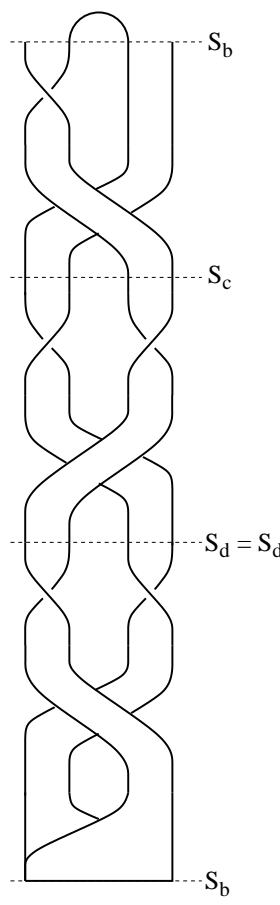

(c) Regular direct mixer from $\mathrm{S}_{b}$

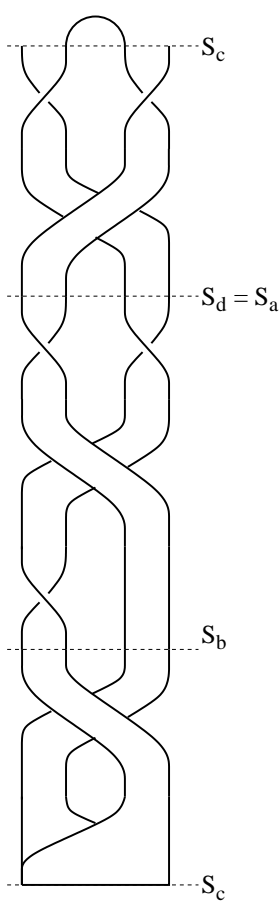

(d) Regular direct mixer from $\mathrm{S}_{c}$

Figure 7. The direct template describing the asymmetric chaotic attractor $\mathcal{A}$ solution to the system (10). The corresponding regular mixers built from section $\mathrm{S}_{a}, \mathrm{~S}_{b}$ and $\mathrm{S}_{c}$ are shown. The mixer $\mathcal{M}_{d}$ is equivalent to the mixer $\mathcal{M}_{\mathcal{A}_{a}}$. Parameter value: $\alpha=2.0645$.

\section{The companion attractor by the inversion symmetry}

Due to the inversion symmetry, the asymmetric chaotic attractor $\mathcal{A}$ has a companion attractor $\overline{\mathcal{A}}=\Gamma \cdot \mathcal{A}$ (Fig. 8) which coexists in the phase space as already mentioned. The topology of the attracor $\overline{\mathcal{A}}$ is constrained by the symmetry properties as detailed below. For the sake of simplicity, the symmetric object of an object $\mathcal{O}$ will be designated by $\overline{\mathcal{O}}$. We will apply the same notation to the linking matrices, the linking matrix $\bar{L}_{\mathcal{O}}=L_{\overline{\mathcal{O}}}$ thus designating the linking matrix of the symmetric companion of the object $\mathcal{O}$ described by the linking matrix $L_{\mathcal{O}}$. 
Systematic template extraction from chaotic attractors. I genus-one attractors with an inversion symmetry 13

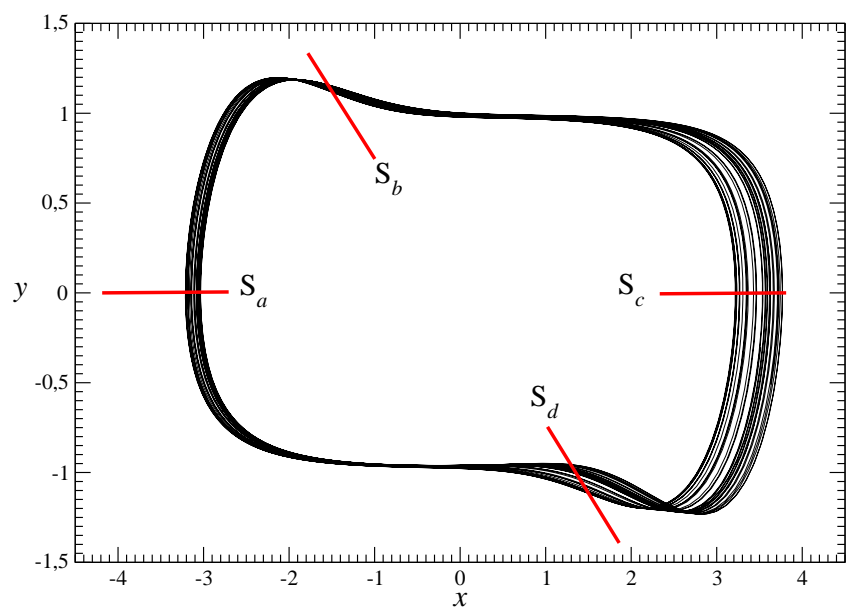

Figure 8. Companion attractor $\overline{\mathcal{A}}$ solution from the symmetric initial conditions $\Gamma \cdot \boldsymbol{x}_{0}$ compared to the attractor $\mathcal{A}$ obtained from initial conditions $\boldsymbol{x}_{0}$. Parameter value: $\alpha=2.0645$.

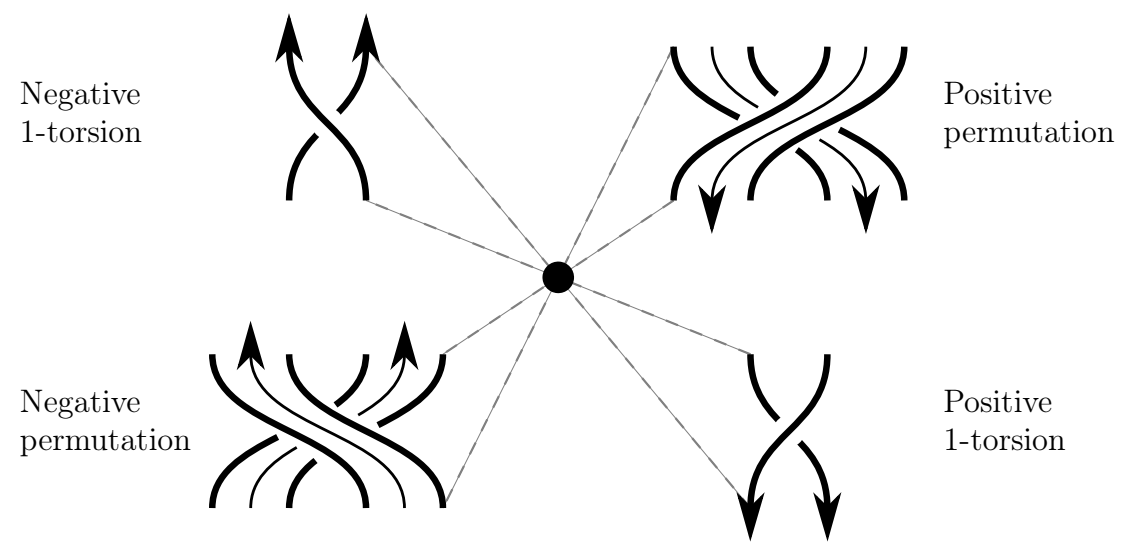

Figure 9. Inversion symmetry applied to a global torsion and a permutation between two branches. (See also Fig 11 of [14])

Before getting the topology of the attractor $\mathcal{A}$, let us start by describing how the dynamical mechanisms, as global torsion, branch permutation and linker, are mapped under an inversion symmetry. Under such a symmetry, a positive global 1-torsion $\mathcal{T}_{+1}$ becomes a negative global 1-torsion $\mathcal{T}_{-1}$ (Fig. 10). In a general way, we have thus

$$
\overline{\mathcal{T}}_{+\eta}=\mathcal{T}_{-\eta},
$$

that is, from the linking matrix point of view,

$$
\bar{T}_{+\eta}=\overline{\left|\begin{array}{ll}
+\eta & +\eta \\
+\eta & +\eta
\end{array}\right|}=\left|\begin{array}{rr}
-\eta & -\eta \\
-\eta & -\eta
\end{array}\right|=T_{-\eta} .
$$

It is now relevant to distinguish a linker without a branch insertion as shown in Fig. 10 from a linker with a branch insertion (the splitting chart does not have any impact 
Systematic template extraction from chaotic attractors. I genus-one attractors with an inversion symmetry 14

on the linking matrix). In the latter case, the linking matrix

$$
T_{-1 \mid}=\left|\begin{array}{cc}
-1 & -1 \\
-1 & -1
\end{array}\right|
$$

of a negative 1-torsion with a branch insertion has for symmetric

$$
\left.\left.\left.\bar{T}_{-1 \mid}=-T_{-1 \mid}-\mid \begin{array}{cc}
0 & +1 \\
+1 & 0
\end{array}\right]=-\left|\begin{array}{cc}
-1 & -1 \\
-1 & -1
\end{array}\right|-\mid \begin{array}{cc}
0 & +1 \\
+1 & 0
\end{array}\right]=\mid \begin{array}{cc}
+1 & 0 \\
0 & +1
\end{array}\right]=T_{+1}
$$

as shown in Fig. 10. In the two previous equations, the vertical bar introduced as subscript means that there is a branch insertion mechanism taken into account in these linking matrices. The second matrix which is substracted to $-T_{-1 \mid}$ is required to preserve the standard insertion convention. Without such a matrix, the branch insertion would not have been in agreement with such insertion convention. Similarly, a positive 1-torsion with a branch insertion $\mathcal{T}_{+1 \mid}$ described by

$$
T_{+1 \mid}=\left|\begin{array}{cc}
+1 & 0 \\
0 & +1
\end{array}\right|
$$

has for symmetric

$$
\left.\left.\bar{T}_{+1 \mid}=-T_{+1 \mid}-\mid \begin{array}{cc}
0 & +1 \\
+1 & 0
\end{array}\right]=-\left|\begin{array}{cc}
+1 & 0 \\
0 & +1
\end{array}\right|-\mid \begin{array}{cc}
0 & +1 \\
+1 & 0
\end{array}\right]=\left|\begin{array}{cc}
-1 & -1 \\
-1 & -1
\end{array}\right|=T_{-1 \mid},
$$

as shown by reading Fig. 10 from the right to the left.

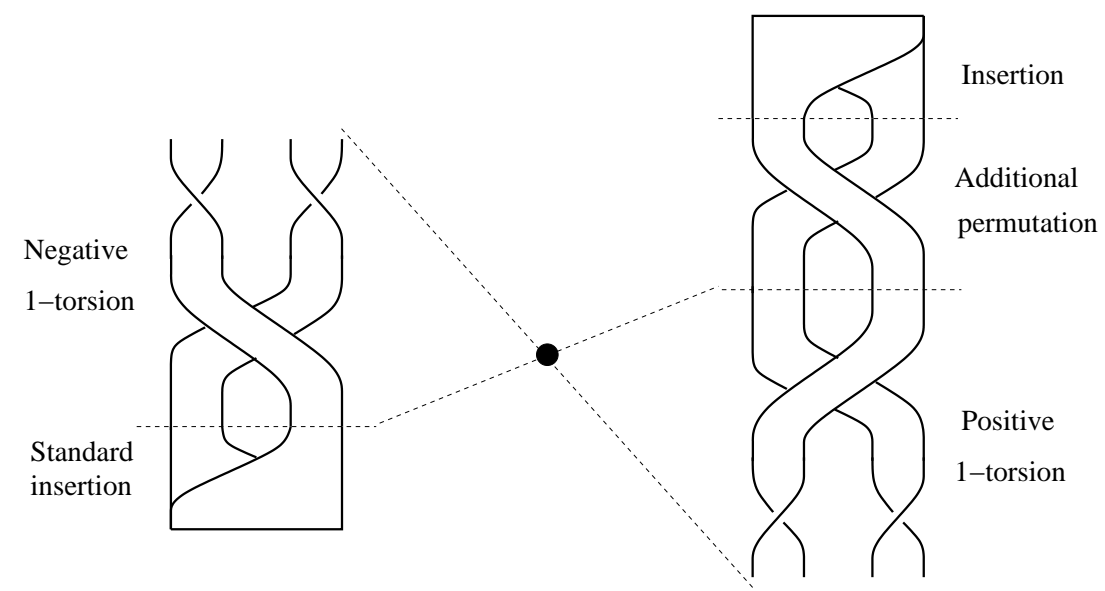

Figure 10. Inversion symmetry applied to a linker made of a torsion $\mathcal{T}_{-1}$ with a branch insertion (left). In the resulting positive torsion $\mathcal{T}_{+1 \mid}$, there is a permutation added to the torsion $\mathcal{T}_{+1}$ to preserve the standard insertion convention.

The rule to obtain the linking matrix $\bar{L}$ of any linker is the same as the rule we introduced for the global torsion, that is,

$$
\bar{L}=-L
$$


Systematic template extraction from chaotic attractors. I genus-one attractors with an inversion symmetry 15
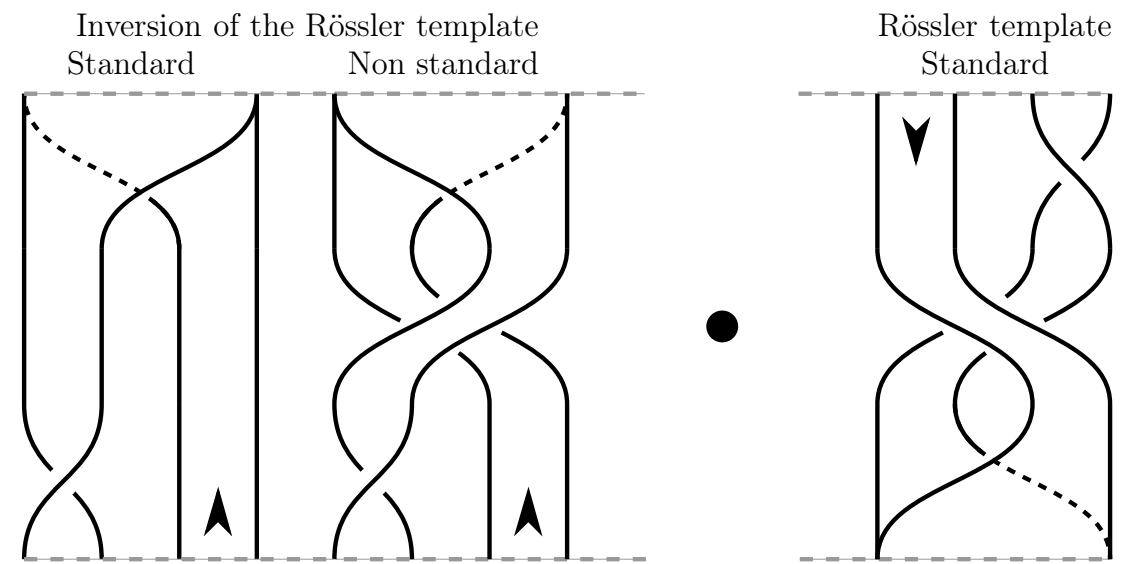

Figure 11. Inversion symmetry applied to the template of the Rössler system. In the resulting template, there is a permutation removed to preserve the standard insertion convention (See also Fig. 10 of [14]).

when there is no branch insertion, and

$$
\left.\bar{L}_{\mid}=-L_{\mid}-\mid \begin{array}{cc}
0 & +1 \\
+1 & 0
\end{array}\right]=-L_{\mid}-\left|\begin{array}{cccc}
0 & +1 & \cdots & +1 \\
+1 & 0 & \ddots & \vdots \\
\vdots & \ddots & \ddots & +1 \\
+1 & \cdots & +1 & 0
\end{array}\right|
$$

when there is a branch insertion and the extension depending of the number of branches, trivial because the order is reversed. This relation illustrates the result already obtained by Letellier and Gouesbet [14] where the inversion symmetry of the Rössler template is the template of the horseshoe mechanism (Fig. 11). Applying these rules to the linking matrix $L_{\mathcal{A}_{a}}$ leads to

$$
L_{\overline{\mathcal{A}}_{c}}=\bar{L}_{\mathcal{A}_{a}}=-\left[\begin{array}{cc}
-1 & -1 \\
-1 & -1
\end{array}|-| \begin{array}{cc}
0 & -1 \\
-1 & -1
\end{array}|-| \begin{array}{cc}
+1 & +1 \\
+1 & +1
\end{array}|-| \begin{array}{cc}
0 & +1 \\
+1 & 0
\end{array}\right]=\left[\begin{array}{cc}
0 & 0 \\
0 & +1
\end{array}\right] .
$$

This is confirmed by the direct template of the attractor $\overline{\mathcal{A}}_{a}$ shown in Fig. 12. Note that section $S_{a}$ is mapped into section $S_{c}$ under the inversion symmetry, and so for sections $\mathrm{S}_{b}, \mathrm{~S}_{c}$ and $\mathrm{S}_{d}$ which are mapped to $\mathrm{S}_{d}, \mathrm{~S}_{a}, \mathrm{~S}_{b}$, respectively.

Now, if we consider the companion attractor $\overline{\mathcal{A}}_{d}$ described starting from section $\mathrm{S}_{d}$, the linking matrix $L_{\mathcal{A}_{b}}$ becomes

$$
L_{\overline{\mathcal{A}}_{d}}=\bar{L}_{\mathcal{A}_{b}}=-\left[\begin{array}{cc}
-1 & -1 \\
-1 & 0
\end{array}|-| \begin{array}{cc}
+1 & +1 \\
+1 & +1
\end{array}|-| \begin{array}{cc}
-1 & -1 \\
-1 & -1
\end{array}|-| \begin{array}{cc}
0 & +1 \\
+1 & 0
\end{array}\right]=\left[\begin{array}{cc}
+1 & 0 \\
0 & 0
\end{array}\right]
$$

when the previous rule is applied. We have thus

$$
L_{\overline{\mathcal{A}}_{a}}=L_{\overline{\mathcal{A}}_{b}}^{p}=L_{\overline{\mathcal{A}}_{c}}^{p}=L_{\overline{\mathcal{A}}_{d}},
$$

which is symmetric to Eq. (18). By a systematic procedure to be applied to the linking matrices as previously introduced, it is thus possible to obtain the linking matrix $L_{\overline{\mathcal{A}}}$ of the attractor $\overline{\mathcal{A}}$ which is the image under an inversion symmetry of an attractor $\mathcal{A}$ described by a linking matrix $L_{\mathcal{A}}$. 


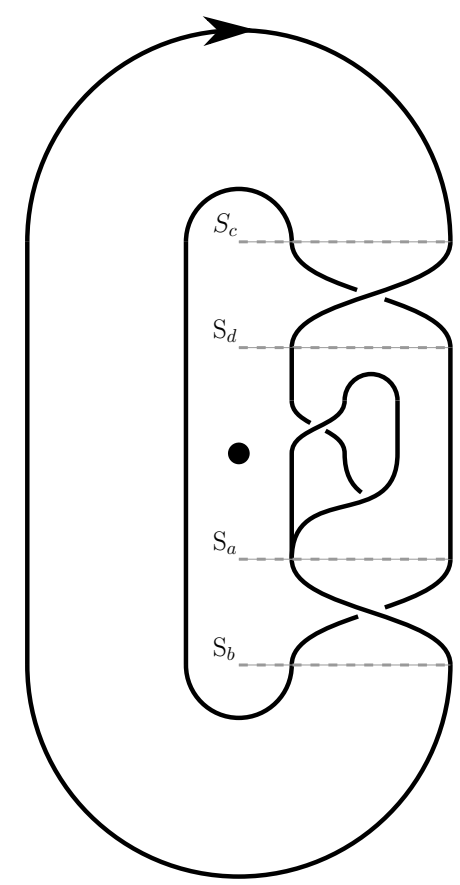

(a) Non regular direct template

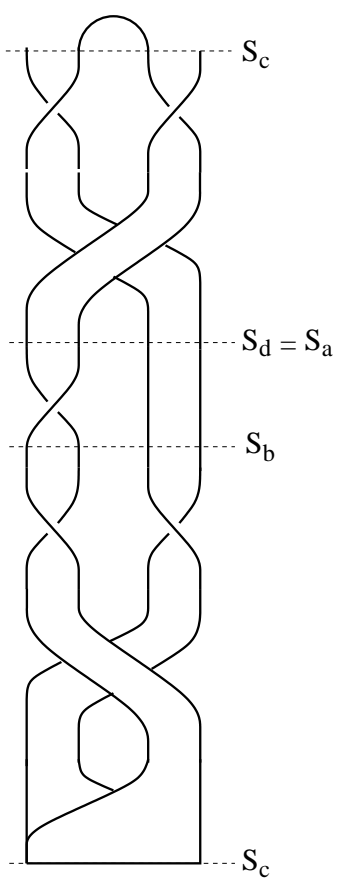

(b) Regular direct template

Figure 12. Direct templates of the companion attractor $\bar{A}_{a}$ solution to the simplest equivariant jerk system (8). Parameter value: $\alpha=2.0645$.

\section{Conclusion}

A template, reduced or direct, can be divided into two parts, one which is trivial where the flow is everywhere locally parallel, and one where there are a splitting chart, torsions (local or/and global), permutations and a branch insertion through stretching and squeezing mechanisms. We described the second part in a general way by a linker associated with a linking matrix. In this work, we used the standard insertion convention. We proposed to represent the template in such a way as the external boundary undergoes in the clockwise direction as used for the bounding tori introduced by Tsankov and Gilmore. The linker is thus always drawn at the right side of the singular point surrounded by the flow. There is thus a unique reduced template for a given linking matrix. To describe the direct template, a set of linking matrices is required to explicitly describe all the identified mechanisms.

Typically, a linker is schemed by a splitting chart allowing to distinguish the different branches which can be easily identified according to the critical points of a first-return map to a Poincaré section. Then, depending on the attractor and the Poincaré section chosen, the linker can be made of successive global torsions and linkers, global torsions being a particular type of linkers. According to this, a linker can be made of linkers. The linker sketching all mechanisms producing the chaotic attractor under study is terminated by the insertion of the branches into the trivial part of the template. A linker beginning with a splitting chart and ending with a branch insertion is a mixer (thus a particular type of linker), its name chosen for its mixing properties leading to chaotic solutions. We introduced the addition law for 
combining the linking matrices describing these global torsions and linkers. Such a law is working until the number of branches is kept constant. From this addition law, it was possible to deduce the general rule for getting the linking matrix describing the linker $\overline{\mathcal{L}}$ image of the linker $\mathcal{L}$ under an inversion symmetry. This was a required step before adressing the topology of attractor globally invariant under symmetry properties as the double-scroll attractor produced by the Chua circuit.

In order to do that in a very general way, a "multiplicative" law, postponed for future works, is required for combining linker of different dimensions. The additive law developed in this paper was the first necessary step to complete before.

\section{Acknowledgments}

The authors thank Robert Gilmore for stimulating discussions while this manuscript was in preparation.

\section{References}

[1] O. E. Rössler, An equation for continuous chaos, Physics Letters A, 57 (5), 397-398, 1976.

[2] E. N. Lorenz, Deterministic nonperiodic flow, Journal of the Atmospheric Sciences, 20, 130141, 1963.

[3] O. E. Rössler, Chaos in abstract kinetics: two prototypes, Bulletin of Mathematical Biology, 39, 275-289, 1977.

[4] L. 0. Chua, M. Komuro \& T. Matsumoto, The double scroll family, IEEE Transactions on Circuit and Systems, 33 (11), 1073-1118, 1986.

[5] D. Li, A three-scroll chaotic attractor, Physics Letters A, 372 (4), 387-393, 2008.

[6] C. Letellier \& R. Gilmore, Poincaré sections for a new three-dimensional toroidal attractor, Journal of Physics A, 42, 015101, 2009.

[7] N. B. Tufillaro, T. Aвbott \& J. Reilly, An Experimental Approach to Nonlinear Dynamics and Chaos, Addison-Wesley (New York), 1992.

[8] C. Letellier, P. Dutertre \& B. Maheu, Unstable periodic orbits and templates of the Rössler system: toward a systematic topological characterization, Chaos, 5 (1), 271-282, 1995.

[9] G. Boulant, M. Lefranc, S. Bielawski \& D. Derozier, Horseshoe templates with global torsion in a driven laser, Physical Review E, 55 (5), 5082-5091, 1997.

[10] R. Gilmore \& M. Lefranc, The topology of chaos, Wiley, 2002.

[11] C. Letellier, E. Roulin \& O. E. Rössler, Inequivalent topologies of chaos in simple equations, Chaos, Solitons \& Fractals, 28, 337-360, 2006.

[12] T. D. Tsankov \& R. Gilmore, Topological aspects of the structure of chaotic attractors in $\mathbb{R}^{3}$, Physical Review E, 69, 056206, 2004.

[13] C. Letellier, P. Dutertre \& G. Gouesbet, Characterization of the Lorenz system taking into account the equivariance of the vector field, Physical Review E 49 (4), 3492-3495, 1994.

[14] C. Letellier \& G. Gouesbet, Topological characterization of reconstructed attractors modding out symmetries, Journal de Physique II, 6, 1615-1638, 1996.

[15] R. Gilmore \& C. Letellier, The symmetry of chaos, Oxford University Press, 2007.

[16] C. Letellier, P. Dutertre, J. Reizner \& G. Gouesbet, Evolution of multimodal map induced by an equivariant vector field, Journal of Physics A, 29, 5359-5373, 1996.

[17] C. Letellier \& L. A. Aguirre, Required criteria for recognizing new types of chaos: application to the "Cord" attractor, Physical Review E, 85, 036204, 2012.

[18] J.-M. Malasoma, What is the simplest dissipative chaotic jerk equation which is parity invariant?, Physics Letters A, 264 (5), 383-389, 2000.

[19] J. BiRman \& R. Williams, Knotted periodic orbits in dynamical systems -I: Lorenz's equations, Topoloy, 22 (1), 47-82, 1983.

[20] G. B. Mindlin, X.-J. Hou, H. G. Solari, R. Gilmore \& N. B. Tufillaro, Classification of strange attractors by integers, Physical Review Letters, 64 (20), 2350-2354, 1990.

[21] D.J. Cross \& R. Gilmore, Dressed return maps distinguish chaotic mechanisms, Physical Review E, 87 (1), 012919, 2013.

[22] O. E. Rössler, Different types of chaos in two simple differential equations, Zeitschrift für Naturforschung A, 31, 1664-1670, 1976. 
Systematic template extraction from chaotic attractors. I genus-one attractors with an inversion symmetry 18

[23] C. Letellier \& J.-M. Malasoma, Unimodal order in the image os the simplest equivariant chaotic system, Physical Review E, 64, 067202, 2001.

[24] H. Poincaré. Mémoire sur les courbes définies par une équation différentielle, Journal de Mathématiques, III, 8, 251-296, 1882.

[25] P. Collet \& J. P. Eckmann, Iterated maps on the interval as dynamical systems, Progress in Physics, 1, Birkhäuser, Boston, 1980.

[26] HaO BAI Lin, Elementary Symbolic Dynamics and Chaos in Dissipative Systems, World Scientific Publishing, Singapore, 1989. 